\title{
( \\ DOI: 10.3329/jname.v8i1.7388 \\ http://www.bangljol.info \\ COMPARATIVE STUDY BETWEEN FLOWS AROUND SPHERE AND POD USING FINITE VOLUME METHOD
}

\author{
M. M. Karim ${ }^{1}$, M. M. Rahman' ${ }^{2}$ and M. A. Alim ${ }^{3}$ \\ ${ }^{1}$ Department of Naval Architecture and Marine Engineering, Bangladesh University of Engineering and Technology, Dhaka- \\ 1000, Bangladesh. e-mail: mmkarim@name.buet.ac.bd \\ ${ }^{2}$ Postgraduate Student, Department of Naval Architecture and Ocean Engineering, Graduate School of Engineering, Osaka \\ University, Osaka, Japan. e-mail: rahman@naoe.eng.osaka-u.ac.jp \\ ${ }^{3}$ Department of Mathematics, Bangladesh University of Engineering and Technology Dhaka-1000, Bangladesh. e-mail: \\ maalim@math.buet.ac.bd
}

\begin{abstract}
:
Two-dimensional Finite Volume Method (FVM) based on Reynolds-averaged Navier-Stokes (RANS) equations is applied to solve the turbulent viscous flow around sphere and pod. Unstructured grid with boundary layer treatment is constructed around sphere whereas structured grid is generated around pod. Spalart-Allmaras (S-A) and Shear Stress Transport (SST) k- $\omega$ turbulence models are used for sphere but SST $k$ - $\omega$ turbulence model is used only for pod to solve turbulent viscous flows at Reynold's number of $5 \times 10^{6}$ and $3 \times 10^{6}$ respectively. The numerical results in terms of the skin friction coefficient, pressure coefficient and drag coefficient are shown either graphically or in the tabular form. Velocity vectors as well as contour of pressure and velocity distribution are also displayed. Finally, the comparative study between flows around sphere and pod is done.
\end{abstract}

Keywords: Finite volume method (FVM), axisymmetric body of revolution, sphere, pod, viscous drag, CFD, turbulence model.

\section{Introduction}

Computational Fluid Dynamics (CFD) is one of the branches of fluid mechanics that uses numerical methods and algorithms to solve and analyze problems involving fluid flows. With the advent of more powerful computers and more comprehensive computer codes, CFD has come to the forefront as a legitimate and effective research tool. CFD analysis can be much more cost effective compared to experimental models since changes can be made quickly and easily to almost any characteristic of the simulation. Only a few base cases are compared with experiment for validation purposes and then it is assumed safe to say that the other CFD simulations in that range are valid. Even if some simulations are analyzed both experimentally and numerically, CFD is still beneficial since it has the ability to offer more information to researchers about the flow.

In CFD, the Finite Volume Method (FVM) is one of the best ways to solve the fluid structure interaction problem. The features of the Finite Volume Method were discussed in details by Versteeg and Malasekera (1995). This method discretises the integral form of the governing equations directly in physical space. The resulting statements express the exact conservation of relevant properties for each finite cell volume. As this method works with the cell volumes and not the grid intersection points, both structured and unstructured meshes can be used. The scheme has the advantage that boundary conditions are more easily applied since the variables are known on all boundaries.

In the last two decades, different areas of incompressible flow modeling including grid generation techniques, solution algorithms and turbulence modeling, and computer hardware capabilities have witnessed tremendous development. Simulation of underwater hydrodynamics continues to be based on the solution of the Reynoldsaveraged Navier-Stokes (RANS) equations. Various researchers used turbulence modeling to simulate flow around axisymmetric bodies since late seventies. Patel and Chen (1986) made an extensive review of the simulation of flow past axisymmeric bodies. Choi and Chen (1990) gave calculation method for the solution of RANS equation, together with $k-\varepsilon$ turbulence model. The flow around the sphere is a basic study in the field of fluid dynamics. The basic structure of the flow past a sphere was experimentally investigated using a variety of

1813-8235 (Print), 2070-8998 (Online) ( 2011 ANAME Publication. All rights reserved. $\quad$ Received on: March 2011


approaches, including flow visualization by Achenbach (1972), Taneda (1978), Bakic (2002), etc. Recent timeaccurate computations of laminar and turbulent flow around spheres using different methods are reported by many researchers; among them the work of Kalro and Tezduyan (1998), Gregory (2000) and Karim et al. (2009A) are remarkable.

The study of flow around pod is of increasing importance due to extensive use of podded propulsors. The podded propulsion systems are becoming increasingly popular in modern commercial marine vessels on account of the increase of their efficiency. Considering its importance a lot of study was done by many researchers. The result of the present paper is compared with that of Gupta (2004). Numerical prediction of the performance of podded propulsors and ducted propellers by Kinnas et al. (2004) are also significant. Karim et al. (2009-B) studied the turbulent viscous flow around submarine hull using unstructured grid, the body shape was similar to that of pod.

However, effective utilization of CFD for marine hydrodynamics depends on proper selection of turbulence model, grid generation and boundary resolution. Although minimization of drag is one of the most important design criteria, not much effort has been given to determining viscous drag, an important parameter in the development of a new design. This paper presents comparative study between viscous flows around axisymmetric sphere and pod using finite volume method based on Reynolds-averaged Navier-Stokes (RANS) equations.

\section{Theoretical Formulation}

\subsection{Governing equation}

For the incompressible flow past an axisymmetric underwater vehicle hull form, the continuity equation in cylindrical co-ordinate is given by:

$$
\rho\left[\frac{\partial u}{\partial x}+\frac{\partial v}{\partial r}+\frac{v}{r}\right]=S_{m}
$$

where $x$ is the axial coordinate, $r$ is the radial coordinate, $u$ is the axial velocity and $v$ is the radial velocity. The source term $S_{m}$ is the mass added and any user-defined sources.

Also, the axial and radial momentum equations are given by:

$$
\begin{aligned}
& \rho\left[\frac{\partial u}{\partial t}+\frac{1}{r} \frac{\partial}{\partial x}\left(r u^{2}\right)+\frac{1}{r} \frac{\partial}{\partial r}(r u v)\right]=-\frac{\partial p}{\partial x}+\frac{1}{r} \frac{\partial}{\partial x}\left[r \mu\left(2 \frac{\partial u}{\partial x}-\frac{2}{3}(\nabla \cdot \vec{u})\right)\right]+\frac{1}{r} \frac{\partial}{\partial r}\left[r \mu\left(\frac{\partial u}{\partial r}+\frac{\partial u}{\partial x}\right)\right]+F_{x} \\
& \rho\left[\frac{\partial v}{\partial t}+\frac{1}{r} \frac{\partial}{\partial x}(r w)+\frac{1}{r} \frac{\partial}{\partial r}\left(r^{2}\right)\right]=\frac{\partial p}{\partial r}+\frac{1}{r} \frac{\partial}{\partial x}\left[r \mu\left(\frac{\partial v}{\partial x}+\frac{\partial u}{\partial x}\right)\right]+\frac{1}{r} \frac{\partial}{\partial r}\left[r \mu\left(2 \frac{\partial v}{\partial r}-\frac{2}{3}(\nabla \cdot \vec{u})\right)\right]-2 \mu \frac{v}{r^{2}}+\frac{2}{3} \frac{\mu}{r}(\nabla \cdot \vec{u})+F_{r}
\end{aligned}
$$

Where, $p=$ static pressure, $\mu=$ molecular viscosity, $\rho=$ density, $F_{x} \& F_{r}$ are external body forces and

$$
\nabla \cdot \vec{u}=\frac{\partial u}{\partial x}+\frac{\partial v}{\partial r}+\frac{v}{r}
$$

\subsection{Turbulence model}

\subsubsection{The Spalart-Allmaras(S-A) turbulence model}

The Spalart-Allmaras(S-A) turbulence model that is used in this study is a simple one-equation model that solves a modeled transport equation for the turbulent viscosity. This model is designed for wall-bounded flows and gives good results for boundary layers subjected to adverse pressure gradients, much like the flow fields encountered in this study. The transported variable in the Spalart-Allmaras model, ${ }_{v}$, is identical to the turbulent kinematic viscosity except in the near-wall (viscous-affected) region (Fluent Inc. 2005). The transport equation for $\bar{v}$ is

$$
\frac{\partial}{\partial t}(\rho \bar{v})+\frac{\partial}{\partial x_{i}}\left(\rho \bar{v} u_{i}\right)=G_{v}+\frac{1}{\sigma_{\bar{v}}}\left[\frac{\partial}{\partial x_{j}}\left\{(\mu+\rho \bar{v}) \frac{\partial \bar{v}}{\partial x_{j}}\right\}+C_{b 2} \rho\left(\frac{\partial \bar{v}}{\partial x_{j}}\right)^{2}\right]-Y_{v}+S_{\bar{v}}
$$


Where, $G_{v}$ is the production of turbulent viscosity and $Y_{v}$ is the destruction of turbulent viscosity that occurs in the near-wall region due to wall blocking and viscous damping $\sigma_{v}^{-}$and $C_{b 2}$ are constants and $v$ is the molecular kinematic viscosity. To obtain the modified turbulent viscosity, $v$, for the Spalart-Allmaras model from the turbulence intensity, $I$ and length scale, $l$, the following equation can be used:

$v=\sqrt{\frac{3}{2}} U_{\text {avg }} I l ; \quad$ Where, $l=0.07 \times L$ and $I=0.16(\mathrm{Re})^{-\frac{1}{8}}$

In this model the constants are considered as:

$C_{b i}=0.1355, C_{b 2}=0.622 C_{v 1}=7.1, \quad C_{w 2}=0.03, \quad C_{w 3}=2, \quad P_{r}=0.667$

\subsubsection{The Shear-Stress Transport (SST) $k$ - $\omega$ model}

The SST $k$ - $\omega$ turbulence model is a two-equation eddy-viscosity model developed by Menter (1994) to effectively blend the robust and accurate formulation of the $k-\omega$ model.

The shear-stress transport (SST) $k-\omega$ model is so named because the definition of the turbulent viscosity is modified to account for the transport of the principal turbulent shear stress. The use of a $k-\omega$ formulation in the inner parts of the boundary layer makes the model directly usable all the way down to the wall through the visous sub-layer; hence the SST $k$ - $\omega$ model can be used as a Low-Re turbulence model without any extra damping functions. The SST formulation also switches to a $k-\varepsilon$ behaviour in the free-stream and thereby avoids the common $k-\omega$ problem that the model is too sensitive to the inlet free-stream turbulence properties. It is this feature that gives the SST $k$ - $\omega$ model an advantage in terms of performance over both the standard $k$ - $\omega$ model and the standard $k-\varepsilon$ model. Other modifications include the addition of a cross-diffusion term in the $\omega$ equation and a blending function to ensure that the model equations behave appropriately in both the near-wall and farfield zones. Transport equations for the SST $k$ - $\omega$ model are given by:

$$
\begin{aligned}
& \frac{\partial}{\partial t}(\rho k)+\frac{\partial}{\partial x_{i}}\left(\rho k u_{i}\right)=\frac{\partial}{\partial x_{j}}\left(\Gamma_{k} \frac{\partial k}{\partial x_{j}}\right)+\tilde{G}_{k}-Y_{k}+S_{k} \\
& \frac{\partial}{\partial t}(\rho \omega)+\frac{\partial}{\partial x_{i}}\left(\rho \omega u_{i}\right)=\frac{\partial}{\partial x_{j}}\left(\Gamma_{\omega} \frac{\partial \omega}{\partial x_{j}}\right)+G_{\omega}-Y_{\omega}+D_{\omega}+S_{\omega}
\end{aligned}
$$

In these equations, $\widetilde{G}_{k}$ represents the generation of turbulence kinetic energy due to mean velocity gradients, $G_{\omega}$ represents the generation of $\omega, \Gamma_{k}$ and $\Gamma_{\omega}$ represent the effective diffusivity of $k$ and $\omega$, respectively, $Y_{k}$ and $Y_{\omega}$ represent the dissipation of $k$ and $\omega$ due to turbulence, $D_{\omega}$ represents the cross-diffusion term, $S_{k}$ and $S_{\omega}$ are userdefined source terms.

\subsection{Boundary conditions}

Since the geometry of an axisymmetric underwater body is, in effect, a half body section rotated about an axis parallel to the freestream velocity, the bottom boundary of the domain is modeled as an axis boundary. Additionally, the left and top boundaries of the domain are modeled as velocity inlet, the right boundary was modeled as an outflow boundary, and the surface of the body itself was modeled as a wall.

\subsection{Viscous drag}

The viscous drag of a body is generally derivable from the boundary-layer flow either on the basis of the local forces acting on the surface of the body or on the basis of the velocity profile of the wake far downstream. The local hydrodynamic force on a unit of surface area is resolvable into a surface shearing stress or local skin friction tangent to the body surface and a pressure normal to the surface. The summation over the whole body surface of the axial components of the local skin friction and of the pressure gives, respectively, the skin-friction $\operatorname{drag} D_{f}$ and the pressure drag $\mathrm{D}_{\mathrm{p}}$ which for a body of revolution in axisymmetric flow become

$$
D_{f}=2 \pi \int_{0}^{x_{e}} r_{w} \tau_{w} \cos \alpha d x D_{p}=2 \pi \int_{0}^{x_{e}} r_{w} p \sin \alpha d x
$$

where, $r_{w}$ is the radius from the axis to the body surface, $\alpha$ is the arc length along the meridian profile, and $x_{e}$ is the total arc length of the body from nose to tail. The sum of the two drags then constitutes the total viscous drag, $D$ or $D=D_{f}+D_{p}$. The drag coefficient $C_{D}$ and the pressure coefficient, $C_{p}$ based on some appropriate reference area $A$ are given by: 


$$
C_{D}=\frac{D}{0.5 \rho U_{\infty}^{2} A} \text { and } C_{p}=\frac{p-p_{\infty}}{0.5 \rho U_{\infty}^{2} A}
$$

where, $p_{\infty}$ is pressure of free stream and $U_{\infty}$ is free stream velocity.

\section{Grid Generation}

\subsection{Unstructured grid around sphere}

It is ensured that in the selected model, the numerical results would be accurate and that the problem would be solvable in a reasonable amount of time. The computational domain is extended ten times the sphere diameter in fore, aft and vertical direction of the sphere.

For the purposes of unstructured grid construction, the computational domain for sphere model is divided into two regions: the boundary layer region and the free stream region. Dividing the domain in this fashion is a common practice in problems where the effects of the viscous boundary layer that forms on the body are expected to significantly affect the flow field and where enhanced grid resolution in the vicinity of the boundary layer is important. The growth factors are chosen to increase the resolution of the meshes at the base of the boundary layers (where flow parameter gradients are largest) while still maintaining high grid resolution, low cell skewness at the top of the boundary layers, and a total boundary layer mesh thickness of approximately 3 $\mathrm{cm}$. Low skewness is important to ensure similar cell proportions between outer boundary layer cells and neighboring free stream region cells.

Meshing of the free stream regions took place in two steps. First, the edges of the regions are meshed, and then, using the edge meshes, the interiors of the regions (or faces) are meshed. Since boundary layer meshing has already been performed, only the axis boundary, inlet, outlet, and top edges have to be meshed. Comparatively course meshes are specified on the exterior (inlet, outlet, and top) boundaries due to the expected lack of large flow property fluctuations (and thus low grid densities) in those regions. For the purpose of grid construction, the computational domain is divided into three faces: Middle face, Front face and Rear face.

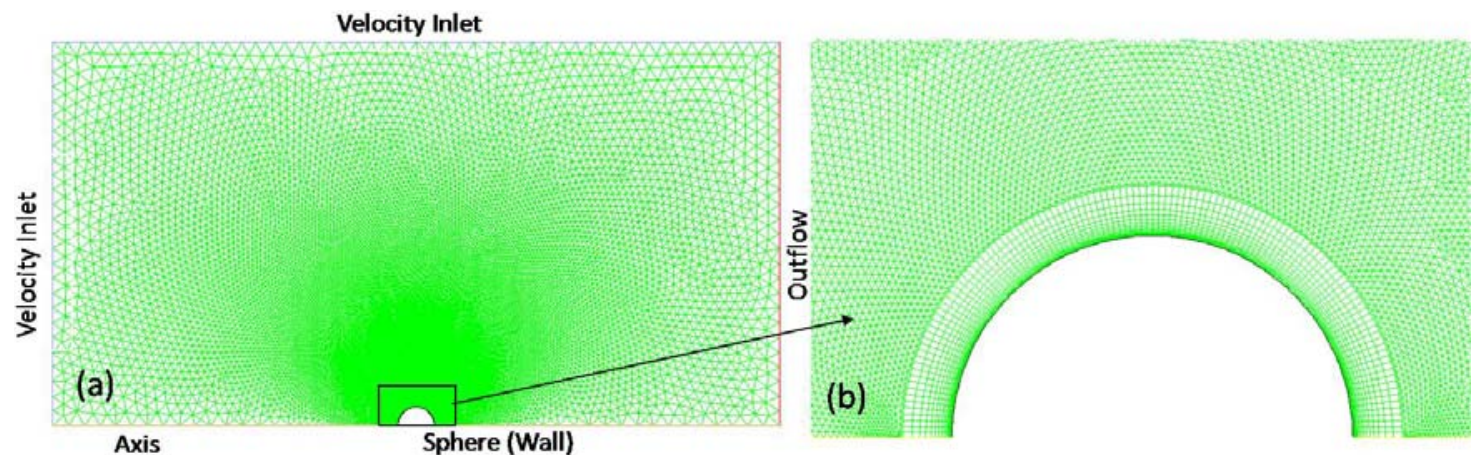

Fig. 1: (a) Axisymmetric sphere unstructured grid with boundary conditions; (b). Close up view near boundary layer of sphere

First the edges are meshed, and then the interior of the domains are meshed using pave meshing scheme. The pave scheme creates an unstructured grid of mesh elements, which is particularly desirable for its applicability to a wide range of face geometries, its ability to deal with irregularly shaped interiors, and its ease of use. There is no restriction on mesh node spacing imposed by the pave scheme since only triangular face elements are used. More cells are constructed near the surface of the sphere to tackle the high velocity gradient in the boundary layer region of the viscous flow. Fig. 1(a) shows the grid for the axisymmetric sphere, which is symmetric about the axis of rotation. Also, Fig. 1(b) shows the close up view that visualizes the boundary layer clearly.

\subsection{Structured grid around pod}

The geometry of the pod is considered for being consistent with the numerical work of Gupta (2004). The computational domain extend 1.5 body lengths upstream of the leading edge of the pod, 1.5 body length above 
the body surface and two body lengths from the trailing edge The solution domain is ensured large enough to capture the entire viscous interaction and the wake development. The mesh generation procedure for this work is based on trial and error. This needs to be fine enough to produce grid independent solutions but coarse enough to have a reasonable computation time. Fig. 2 shows the grid for the axisymmetric model of pod hull, which is symmetric about the axis of rotation. More cells are concentrated near the leading edge and trailing edge of the hull to capture the stagnation points. Since the fine resolution of grid points is not required near the inflow and outflow boundaries, an expansion ratio is used from the leading edge of the body to the inflow boundary and from the trailing edge to the outflow boundary. The node distribution with the expansion ratio of pod is given in Table 1.

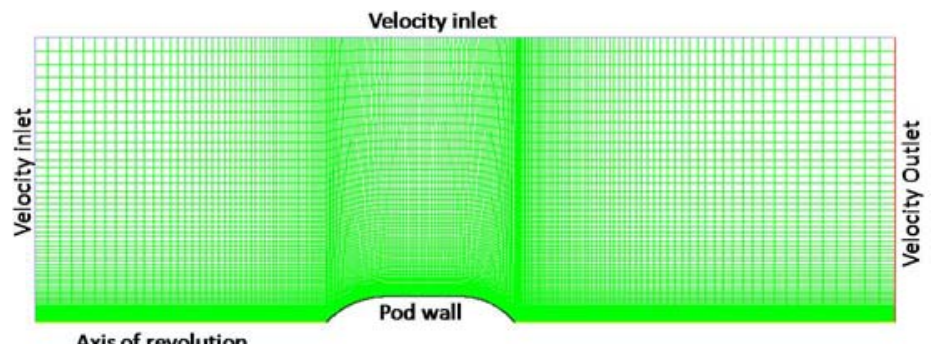

Fig. 2: 2D- Axisymmetric pod grid with boundary condition

Table 1: Axisymmetric pod boundary node spacing distributions

\begin{tabular}{|l|l|l|l|l|l|l|l|}
\hline \multicolumn{2}{|c|}{ Front face } & \multicolumn{2}{c|}{ Middle face } & \multicolumn{3}{c|}{ Rear face } & \multicolumn{2}{c|}{ Normal to the axis } \\
\hline $\begin{array}{l}\text { No. of } \\
\text { Nodes }\end{array}$ & $\begin{array}{l}\text { Growth } \\
\text { factor }\end{array}$ & $\begin{array}{l}\text { No. of } \\
\text { Nodes }\end{array}$ & $\begin{array}{l}\text { Growth } \\
\text { factor(both } \\
\text { direction) }\end{array}$ & $\begin{array}{l}\text { No. of } \\
\text { Nodes }\end{array}$ & $\begin{array}{l}\text { Growth } \\
\text { factor } \\
\text { Nodes }\end{array}$ & $\begin{array}{l}\text { No. of } \\
\text { factor }\end{array}$ \\
\hline 50 & 1.04 & 80 & 1.02 & 70 & 1.04 & 70 & 1.05 \\
\hline
\end{tabular}

\section{Methodology}

For all of the grids generated in this study, the segregated solver formulation is used. This approach solves the continuity, momentum, and energy equations sequentially as opposed to simultaneously. A finite volume method (Versteeg and Malasekera, 1995) is employed to obtain a solution of the Reynold's averaged NavierStokes equations. The coupling between the pressure and velocity fields was achieved using PISO algorithm (Barton, 1998). A second order upwind scheme was used for the convection and the central-differencing scheme for diffusion terms.

In every case, after the flow is successfully initialized at the boundary, the solution is iterated until the convergence is attained. Convergence is declared if the x-velocity, y-velocity, and continuity residuals all dropped below 0.001 .

\section{Results and Discussion}

\subsection{Flow around sphere}

In the case of axisymmetric turbulent flow around sphere, the computed results are compared to Achenbach's (1972) experimental data. Achenbach's experiment is performed at a transcritical Reynolds number where the flow is considered fully turbulent and thus can be directly compared to the turbulent computational models. In the present study, the turbulent flow is well captured with Spalart-Allmaras (S-A) and shear stress transport $k$ - $\omega$ turbulence model at $\operatorname{Re}=5 \times 10^{6}$.

The predicted pressure coefficient over the surface of the sphere is shown in Fig. 3. The computed results are very close to the Achenbach's experimental values. Fig. 4 shows computed values of skin friction coefficient over the sphere. In this case, the computed skin friction coefficient curves does not track well with Achenbach's data forward of the separation point. However, the general trends of the curves are the same. However, the discrepancies between actual and computed $C_{f}$ curves are not expected to greatly affect the reliability of the total 
drag prediction since skin friction drag accounts only for $9.6 \%$ of the total drag in this case. Here, the computed $C_{p}, C_{f}$ and $C_{D}$ are $0.1468,0.0156$ and 0.1625 respecyively. Fig. 5(a) shows the velocity vectors around sphere. The separated region and vortex shedding are clearly visible in the close up view near wall as shown in Fig. 5(b). Table 2 shows the angular position of separation points as well as the percentage of difference from experimental values. The numerical predictions of separation point matched Achenbach's experimental data well.

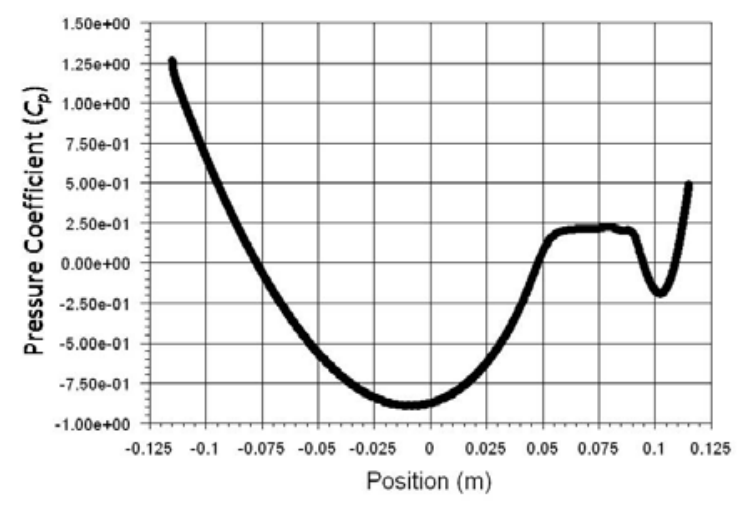

Fig. 3: Plot of pressure coefficient on the surface of sphere at $\operatorname{Re}=5 \times 10^{6}$

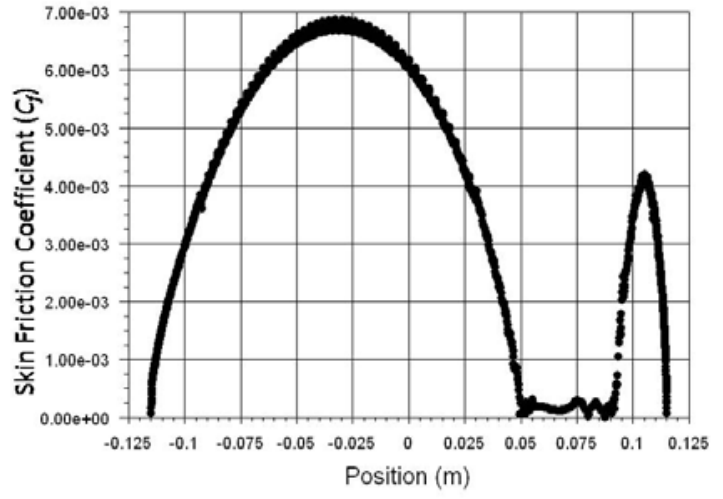

Fig. 4: Plot of skin friction coefficient on the surface of sphere at $\mathrm{Re}=5 \times 10^{6}$

Table 3 shows values of drag coefficient predicted by two turbulence models and also experimental values measured by Achenbach (1972). Percentage of difference between the numerical and experimental values is also included. The small discrepancy between Achenbach's drag coefficient and the numerically computed drag coefficient may be due to Reynolds number mismatch.

Table 2: Angle of separation for axisymmetric turbulent flow around sphere

\begin{tabular}{|c|c|c|}
\hline & $\begin{array}{c}\text { Separation points } \\
\text { (in degrees ) }\end{array}$ & Percentage of difference \\
\hline Present & 126 & $6.8 \%$ \\
\hline Achenbach's (1972) exp. result & 118 & 6.8 \\
\hline
\end{tabular}

Table 3: Drag coefficient for axisymmetric turbulent flow around sphere

\begin{tabular}{|c|c|c|c|}
\hline & $\begin{array}{c}\text { Spalart-Allmaras } \\
\text { Turbulent model }\end{array}$ & SST $k$ - $\omega$ model & Achenbace's exp. $\left(C_{D}\right)$ \\
\hline Drag coefficient, $C_{D}$ & 0.163 & 0.154 & \multirow{2}{*}{0.18} \\
\hline Percentage of difference & $9 \%$ & $14 \%$ & \\
\hline
\end{tabular}

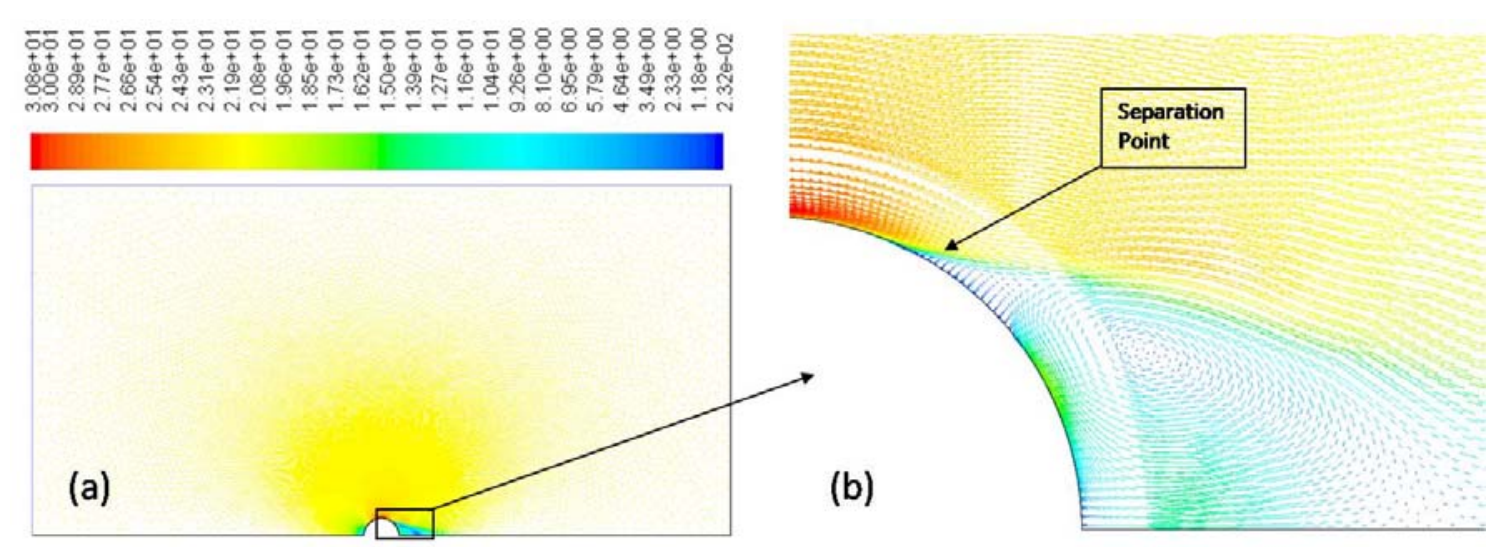

Fig. 5(a): Velocity vectors around sphere (b) Close up view that shows the separation point 


\subsection{Flow around axisymmetric pod}

In this study, only the pod (without strut) is considered. The geometry of the pod is as that of Gupta (2004) for comparison. The Spalart-Allmaras (S-A) turbulence model does not give good results, so only the Shear Stress Transport (SST) $k-\omega$ model is used only for pod. This model captured the turbulent flow around the axisymmetric pod geometry very well at $\mathrm{Re}=3 \times 10^{6}$. The parameters used for the axisymmetric flow solver are given in Table 4.

Table 4: Run parameters for axisymmetric viscous model around pod

\begin{tabular}{|c|c|c|c|}
\hline Solver & 2DDP & Reynolds Number & $3 \times 10^{6}$ \\
\hline Model & SST $k-\omega$ & $\begin{array}{c}\text { Turbulence Kinetic } \\
\text { Energy, } k\end{array}$ & $9.227 \times 10^{-4}$ \\
\hline Density & 1000 & $\begin{array}{c}\text { Specific Dissipation } \\
\text { Rate, } \omega\end{array}$ & 0.2641 \\
\hline Viscosity & 0.001 & $C \mu$ & 0.09 \\
\hline Inflow & $\mathrm{u}=1, \mathrm{v}=0$ & Turbulence Length, $l$ & 0.21 \\
\hline
\end{tabular}

The time step size is selected so that the maximum cell Courant number is very near to unity. In Fig. 6 , the result of $C_{D}$ versus time shows the convergence of the solution.

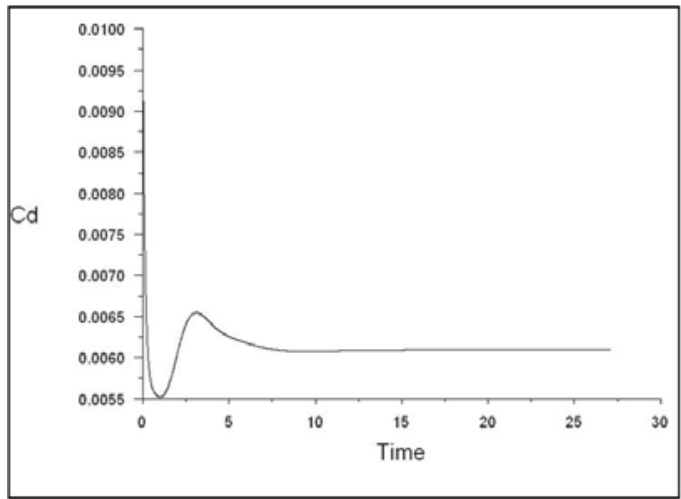

Fig. 6: Time history of drag coefficient for pod

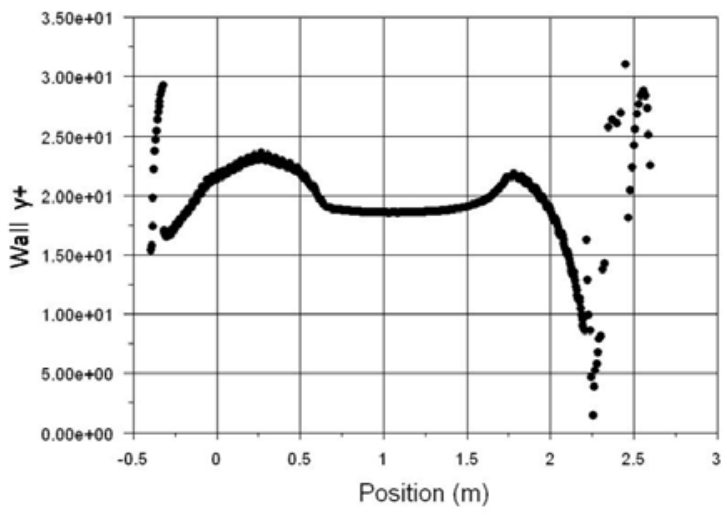

Fig. 7: Plot of $y^{+}$of pod for SST $k$ - $\omega$ model

The minimum grid spacing is generally based on $\mathrm{Y}^{+}$a dimensionless parameter representing a local Reynolds number in the near wall region. It is important for the near wall treatment for turbulent flow. Fig. 7 shows the maximum value of $Y^{+}=25$ (approximate) obtained using SST $k-\omega$ model, which is acceptable when disabling transient flow. Surface pressure coefficient, frictional coefficient and overall volumetric drag coefficients are important quantities for assessing the hydrodynamic efficiency of podded propulsor system. The main focus of this study is to calculate these forces. The computed total drag coefficient with its component at $\operatorname{Re}=3 \times 10^{6}$ is given in Table 5.

Table 5: Total drag coefficient with its component at $\mathrm{Re}=3 \times 10^{6}$ computed on pod hull

\begin{tabular}{|c|c|c|}
\hline Pressure coefficient $\left(C_{p}\right)$ & Frictional coefficient $\left(C_{f}\right)$ & Drag coefficient $\left(C_{D}\right)$ \\
\hline $2.199 \times 10^{-3}$ & $3.89 \times 10^{-3}$ & $6.09 \times 10^{-3}$ \\
\hline
\end{tabular}

From this table, it is observed that, in case of pod the frictional coefficient is also the major portion of total drag like submarine hull due to its long body surface. However, in this case, frictional coefficient is $64 \%$ of total drag coefficient whereas it is $79.5 \%$ in case of submarine (Karim et al., 2009-B). This is happened due to the fact that the pod body has lower $L / D$ ratio $(L / D=3.6)$ than submarine body $(L / D=8.75)$. Small difference in shape at the nose and tail section of the bodies may also be responsible for this. Surface area is one of the important parameters for calculation of skin friction coefficient. In this case, following formula is used to calculate the surface area of pod. 
Area $=\sum_{i}\left(\sqrt{\left(R_{i+1}-R_{i}\right)^{2}+\left(x_{i+1}-x_{i}\right)^{2}} \times\left(R_{i+1}+R_{i}\right) \times \pi\right)$

Where, $R_{i}$ are the radii at corresponding axial location $\mathrm{x}_{i}$.

Table 6 shows the comparison of the computed frictional coefficient with results of Gupta (2004) and Blasius (1908). It is observed from the table that the computed result shows better agreement than that of Gupta.

Table 6: The frictional coefficient of pod

\begin{tabular}{|c|c|c|}
\hline Present Result $\left(C_{f}\right)$ & $\begin{array}{c}\text { Gupta's result }\left(C_{f}\right) \\
(\text { Calculated by RSM model) }\end{array}$ & Blasius theoretical result $\left(C_{f}\right)$ \\
\hline $3.89 \times 10^{-3}$ & $5.0838 \times 10^{-3}$ & $3.207 \times 10^{-3}$ \\
\hline
\end{tabular}

Fig. 8 and Fig. 9 show the pressure coefficient and axial velocity on the pod respectively and both of them agree well with that of Gupta (2004). In this case, the radial velocity is zero everywhere as the flow is simulated at a zero angle of attack. It is known that the easiest way of calculating the separation point is from its wall shear stress.

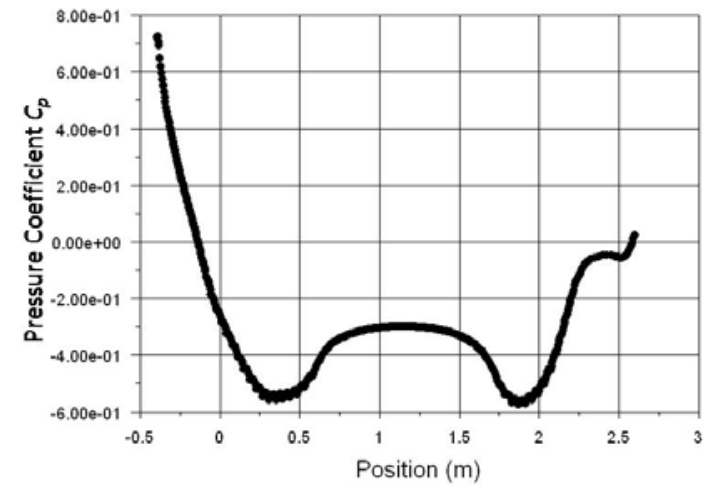

Fig. 8: Plot of pressure coefficient of pod

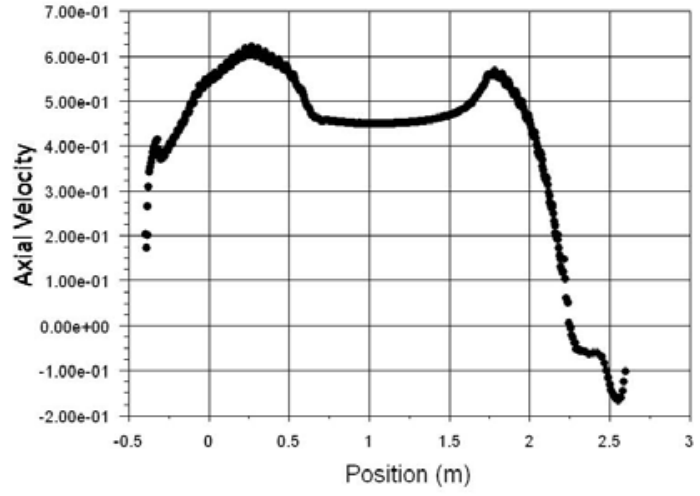

Fig. 9: Plot of axial velocity of pod

At the separated point the wall shear stress become zero. From Fig. 10 it is observed that the wall shear goes to zero but then increase again. This phenomenon demonstrates that boundary layer may have separated and then reattached. This phenomenon demonstrates that boundary layer may have separated and then reattached.

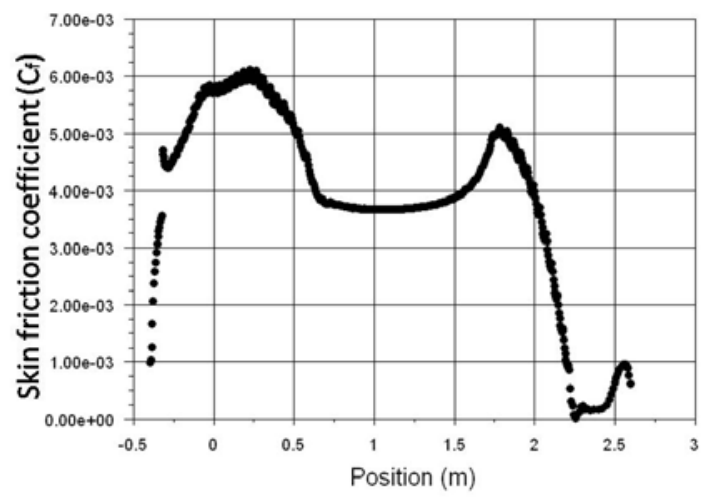

Fig. 10: Plots of skin friction coefficient of pod

Figs. 11-14 show the different flow visualization over the pod hull. As axisymmetric model is used in this study, all of the figures show only half section of the body. The contour of the pressure coefficient around the pod hull is shown in Fig. 11(a) at $\mathrm{Re}=3 \times 10^{6}$. The stagnation point of high pressure at the front tip of the hull, the favorable pressure gradient at the front section and the adverse pressure gradient at the rear section of the hull are clearly shown. 


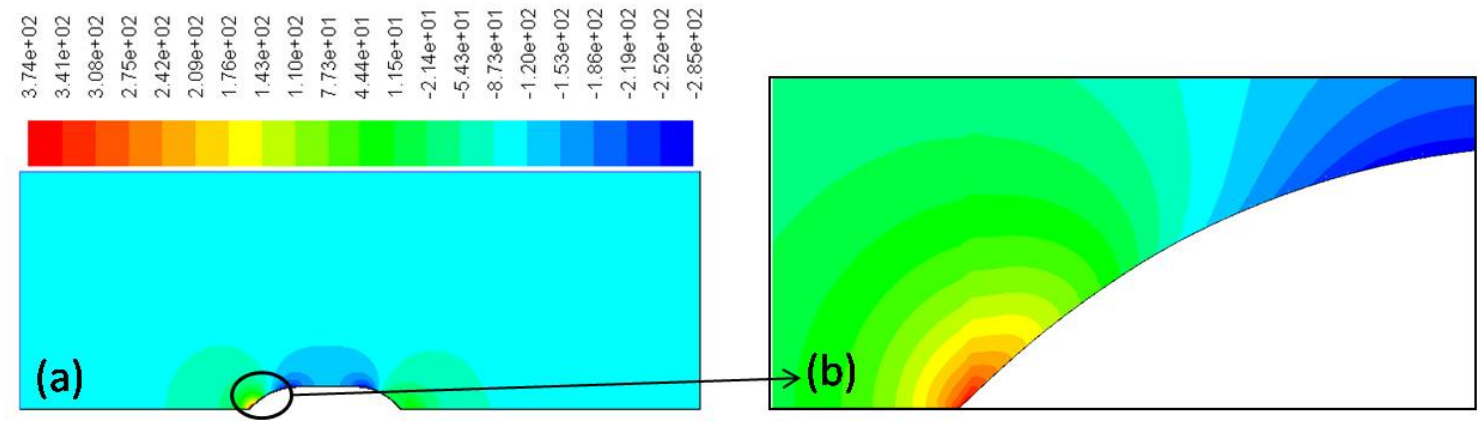

Fig. 11: (a) Contours of pressure coefficient for pod; (b) Close up view near the leading edge

Since the reference pressure is set to zero the pressures shown are relative. Fig. 11 (b) shows a close up of the front section of the hull. Here the stagnation point and the favorable pressure gradient are even more visible (red color).

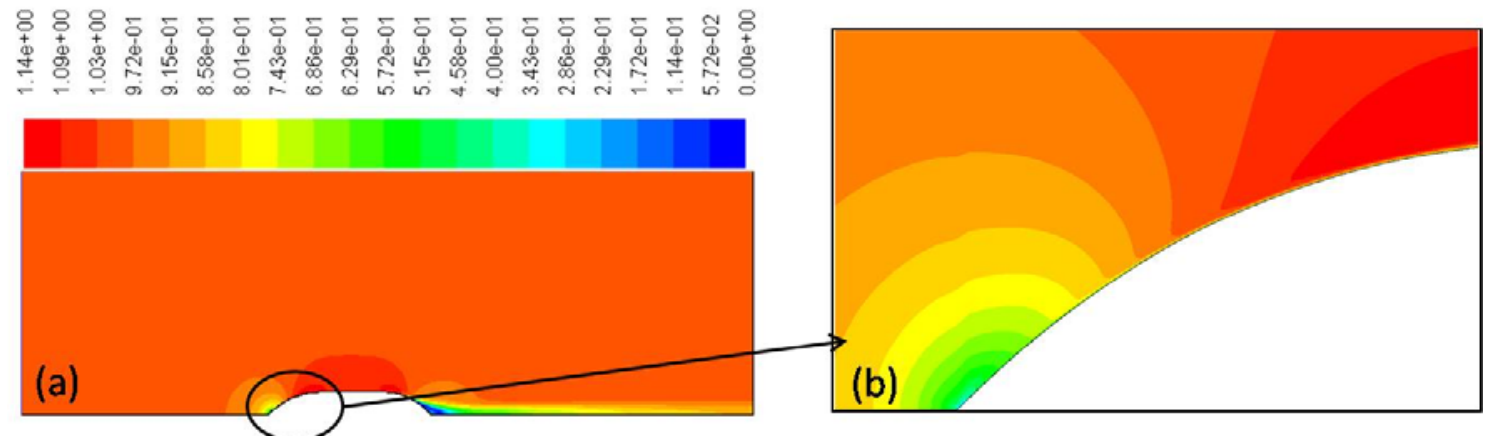

Fig. 12: (a) Contours of velocity magnitude for pod; (b) Close up view near the leading edge

Fig. 12(a) shows the contour of velocity magnitude for the pod hull at $\operatorname{Re}=3 \times 10^{6}$. When compared to the pressure plot it can be seen that the stagnation point of high pressure corresponds to the low velocity point at the front, the favorable pressure gradient in the front section corresponds to a high velocity and the adverse pressure gradient at the rear corresponds to a lower velocity. Fig. 12(b) shows a close up view of the front section of the velocity profile. Here it is apparent by the colors close to the shape that the 'no slip' boundary condition set for the surface of the hull is in effect. It is also more apparent that the stagnation point is actually a stagnation point with zero velocity (the blue region).

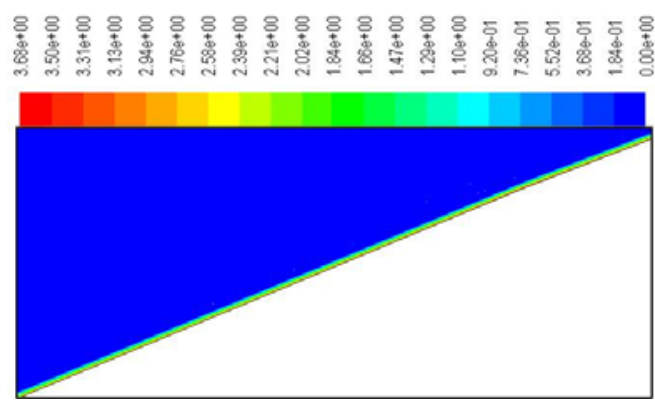

Fig. 13: Contours of wall shear stress for pod
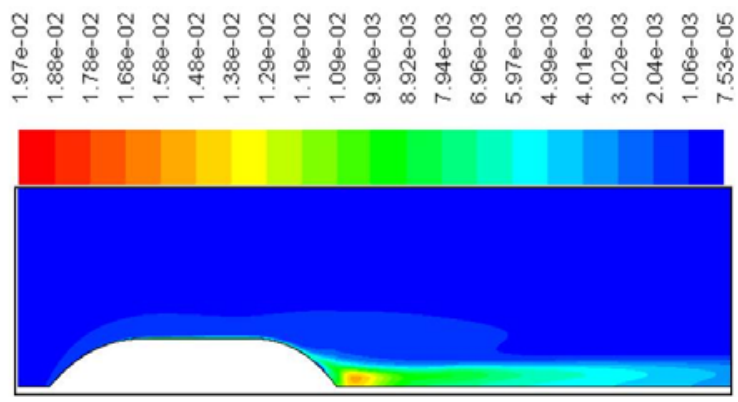

Fig. 14: Contours of turbulence kinetic energy for pod

The wall shear plots are a good indication of the viscous drag over the hull surface. They can also be used to check if there is any separation because the wall shear goes to zero where the boundary layer separates. Viscous effect occurred only on the boundary surface of the body. Large wall shear affect in the favorable pressure gradient area at the front section of the hull. The very peak of the front section has a reduced wall shear, which makes sense physically because there is a reduced flow velocity in this region due to the stagnation point. Fig. 13 shows the boundary surface region closer, which indicates a high shear stress (red color). Finally, turbulence 
kinetic energy is related to the turbulence model used in this simulation. Turbulence originates from the boundaries of a domain, so in this case the only place for turbulence to be generated is from the body, since it is the only region with a no slip condition. Turbulence kinetic energy is a measure of the energy built up as turbulence. In Fig. 14, the kinetic energy is shown to build up as flow passes along the hull and then propagate from the rear.

\section{Conclusion}

The viscous flows around axisymmetric sphere and pod have been computed by Finite Volume Method (FVM) based on Reynold's averaged Navier-Stokes equations. From the above mentioned study, following conclusions can be drawn:

a) Drag coefficients and separation angle computed by the unsteady axisymmetric flow solver agrees well with experimental results.

b) Spalarat-Allmaras (S-A) turbulence model show better performance than shear stress transport (SST) $k$ $\omega$ model in case of sphere $(L / D=1)$ whereas the (SST) $k-\omega$ turbulence model computes the value of drag coefficient very well in case of axisymmetric pod $(L / D=3.6)$.

c) The frictional coefficient is $9.6 \%$ of the total drag coefficient in case of sphere whereas it is $64 \%$ in case of pod.

d) The shear stress transport $k$ - $\omega$ model shows better performance than other turbulence model for bodies where the frictional force dominates the total drag force.

\section{REFERENCES}

Achenbach, E., (1972): Experiments on the Flow past Spheres at Very High Reynolds Numbers, Journal of Fluid Mechanics, vol. 54.3, pp. 565-575. doi:10.1017/S0022112072000874

Bakic, V., (2002): Experimental Investigation of Turbulent Flows around a Sphere, Ph.D. Thesis, TUHH Hamburg, Germany.

Barton, I. E., (1998): Comparison of SIMPLE- and PISO-Type Algorithms for Transient Flows, International Journal for Numerical Methods in Fluids, vol. 26, 4, pp. 459-483. doi:10.1002/(SICI)1097-0363(19980228)26:4<459::AIDFLD645>3.0.CO;2-U

Blasius, H., (1908): Grenzschichten in Flussigkeiten mit kleiner Reibung, Z. Math. Phys., vol 56, pp. 1-37.

Choi, S. K., Chen, C. J., (1990): Laminar and Turbulent Flows past Two Dimensional and Axisymmetric Bodies, Iowa Institute of Hydraulic Research, IIHR Report 334-II.

Fluent Inc., (2005): FLUENT 6.2 User's Guide.

Gregory, J.S., (2000): Computational Fluid Dynamics Testing for Drag Reduction of an Aircraft Laser Turret, Master's Thesis, Dept. of Air Force, Air Force Institute of Technology, Ohio.

Gupta, A., (2004): Numerical Prediction of Flows around Podded Propulsors, Master's thesis, Department of Civil Engineering, The university of Texas, Austin.

Kalro, V. and Tezduyan, T., (1998): 3D Computation of Unsteady Flow past a Sphere with a Parallel Finite Element Method, Computer Methods in Applied Mechanics and Engineering, vol.151, pp. 267-276. doi:10.1016/S0045-7825(97)00120-5

Kinnas, S. G. H., Gupta, A., and Lee, H., (2004): Numerical Prediction of the Performance of Podded Propulsors and Ducted Propellers, In Proceedings of the 13th Offshore Symposium on The Application of Emerging Technologies Offshore, Houston, Texas.

Karim M., Rahman M. M. and Alim M. A., (2009-A): Computation of Axi-symmetric Turbulent Viscous Flow around Sphere, Journal of Scientific Research, vol.1, no. 2, pp 209-219.

Karim M., Rahman M. M. and Alim M. A., (2009-B): Computation of Turbulent Viscous Flow around Submarine Hull using Unstructured Grid, Journal of Ship Technology. Institute of Naval Architects, India, vol. 5, no. 1, pp. 38-52.

Menter, F. R., (1994): Two-equation Eddy-Viscosity Turbulence Models for Engineering Applications. AIAA Journal, 199432(8):1598-1605. doi:10.2514/3.12149

Patel, V. C., Chen, H.C. (1986): Flow over Tail and in Wake of Axisymmetric Bodies: Review of the State of the Art, Journal of Ship Research, vol. 30, no. 3, pp. 202-314.

Taneda, S., (1978): Visual Observations of the Flow past a Sphere at Reynolds Numbers between 104 and 106, Journal of Fluid Mechanics, vol. 85, pp. 187-192. doi:10.1017/S0022112078000580

Versteeg, H. K., Malalasekera, W., (1995): An Introduction to Computational Fluid Dynamics-the Finite Volume Method, Longman Scientific and Technical, U.K. 